\title{
A Comparative Study of Visual Cues for Adaptive Navigation Support
}

\author{
Roya Hosseini \\ Intelligent Systems Program \\ University of Pittsburgh \\ Pittsburgh, PA 15260 \\ roh38@pitt.edu
}

\author{
Peter Brusilovsky \\ School of Information Sciences \\ University of Pittsburgh \\ Pittsburgh, PA 15260 \\ peterb@pitt.edu
}

\begin{abstract}
Past work in the area of adaptive navigation support assumed no difference between different interface implementations of personalization approaches that are conceptually the same. The goal of this paper was to compare the impact of different implementations of the same adaptive navigation support approach on user perception and performance.
\end{abstract}

\section{Keywords}

adaptive navigation support; link annotation; code examples

\section{DESIGN CHOICES FOR ANS}

Adaptive navigation support (ANS) is a group of technologies that support user navigation in hyperspace by adapting to the goals, preferences, and knowledge of an individual user [1]. Over the years, some efficient ANS approaches were established and evaluated. Many teams suggested different sets of icons to implement conceptually the same personalization approach (such as knowledge-based or prerequisitebased annotations). While each of these efforts was typically evaluated and proven efficient, they implicitly assumed that the choice of icons to implement an adaptation approach does not matter, and that only the approach itself does.

In this paper, we present our attempt to compare different implementations of the same ANS approach in interactive program examples produced by the WebEx system [3]. The original WebEx system has no link annotation, however, more recent versions used simple history-based link annotation: code lines already accessed by the user were annotated with check marks as shown in Figure 1. Our goal was to determine the best knowledge-based annotation approach and to find the best way to combine it with history-based annotation and direct recommendation. We expected that visualizing this information dynamically (i.e., displaying it as a visual cue next to the line) could help users select the most important lines. The design alternatives for icon-based adaptive link annotation in the WebEx examples are as follows.

Permission to make digital or hard copies of part or all of this work for personal or classroom use is granted without fee provided that copies are not made or distributed for profit or commercial advantage and that copies bear this notice and the full citation on the first page. Copyrights for third-party components of this work must be honored. For all other uses, contact the owner/author(s).

HT '16 July 10-13, 2016, Halifax, NS, Canada

(C) 2016 Copyright held by the owner/author(s).

ACM ISBN 978-1-4503-4247-6/16/07.

DOI: http://dx.doi.org/10.1145/2914586.2914615

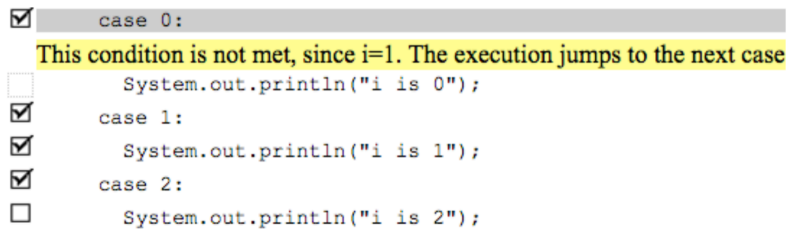

Figure 1: A partial view of an annotated example with a check mark annotation for clicked lines.

Knowledge-based annotation. The first design used a "filling" metaphor displaying icons with different levels of filling to show the knowledge behind each line. This kind of design was explored in the past in $[7,3]$. Five discrete filings were defined from $0 \%$ to $100 \%$, with $25 \%$ increments to represent $0 \%$ to $100 \%$ knowledge behind the line. This design is referred to as $A 1$ (see design $A 1$ in the knowledge-based annotation column of Table 1 ). The second design ( $A 2)$ explored earlier in [6] used different intensities of the green color. As student knowledge increases, the green color of the icons becomes darker (see design $A 2$ in the knowledgebased annotation column of Table 1). The third design (A3), explored earlier in [5], used a gradient that ranged from orange to green colors for the icons relative to the knowledge of the student. As student knowledge increases, the color of the icon changes from dark orange through yellow into dark green (see design $A 3$ in the knowledge-based annotation column of Table 1).

History-based annotation. The first design (B1) borrowed the common Web browser design that changes the color of visited links from blue to purple: the icons next to lines that were viewed by the student are filled with a purple color. Since this history-based annotation must be used jointly with knowledge-based annotation, there were three possible combinations: $B 1(A 1), B 1(A 2)$, and $B 1(A 3)$ shown in column $B 1$ of Table 1 . The second design (B2) followed the approach used in the current version of WebEx (Figure 1): a check mark sign over the bullet to indicate visited lines (see three combinations of this design in column $B 2$ of Table 1).

Recommendations. Two designs were explored for the recommendation of an example line. The first design $(C 1)$ simulates the bold font used, for example, in [2], by increasing the width of the icon border to indicate recommended lines. The second design $C 2$ used a red star as an indicator of recommendation, just as in [4]. Similar to history-based annotation, the recommendation was used with the knowledgebased annotation designs $A 1-A 3$. Columns $C 1$ and $C 2$ of Table 1 illustrate how knowledge-based annotations and recommendations were combined. 
Table 1: Design alternatives for annotation of links in an annotated example

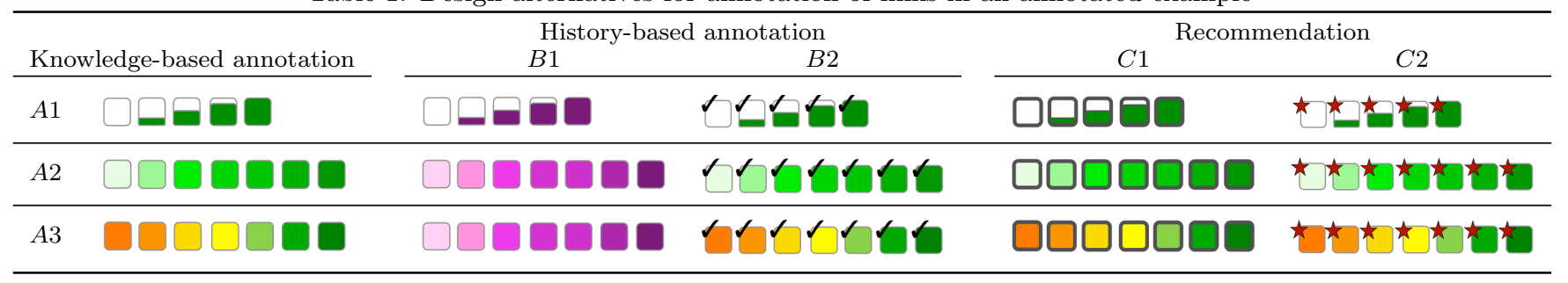

\section{THE STUDY}

We designed and conducted a user study with 31 students at the University of Pittsburgh to assess design alternatives for the three types of icon-based ANS reviewed above. The designs were shown with the full set of icons for each kind of annotation, as shown in Table 1. The subject was asked to provide her/his opinion about each design alternatives by answering a 5-item questionnaire. After that, the subject performed three tasks (Task 1-Task 3 ).

Task 1 provided three code examples annotated according to three different knowledge-based ANS alternatives, i.e., $A 1-A 3$. The subject was asked to circle the lines that showed minimum and maximum knowledge in each example and then she/he had to select the design that made finding the lines with minimum and maximum knowledge easier.

Task 2 provided three annotated code examples and asked the subject to circle already accessed lines. Each example used a combination of knowledge-based annotations $A 1-A 3$ and history-based annotations $B 1-B 2$, which indicated accessed lines. Odd-numbered subjects received combinations $B 1 A 1, B 1 A 3$, and $B 2 A 2$ and even-numbered subjects received combinations $B 1 A 2, B 2 A 1$, and $B 2 A 3$. At the end of the task, the subject had to select the design that made finding the accessed lines easier.

Task 3 provided three annotated code examples and asked the subject to circle the recommended lines in each one. Each example used a combination of knowledge-based annotations $A 1-A 3$, combined with annotations $C 1-C 2$ for showing recommended lines. Odd-numbered subjects received combinations $C 1 A 1, C 1 A 3$, and $C 2 A 2$, and even-numbered subjects received $C 1 A 2, C 2 A 1$, and $C 2 A 3$. At the end of the task, the subject was asked to select the design that made finding the recommended lines easier.

\section{FINDINGS}

The alternative designs were evaluated using data collected from both questionnaires and tasks. Analysis of the questionnaire data showed that the annotation approaches that appeared to be interchangeable were actually considerably different from user prospects. The designs that used filled bullets $(A 1)$ turned out to be significantly better than the design that used different shades of green $(A 2)$ and considerably better than the second-best design $(A 3)$ that used a progression of orange to green colors. The design that annotated an example link with a check mark (B2) was significantly better than the design that used the purple color (B1). Similarly, the design that annotated an example link with a red star $(C 2)$ received significantly higher preference, as compared to the design that used a thick border for the bullet $(C 1)$.

The in-context perceptions of subjects collected during the tasks showed that user preferences changed consider-

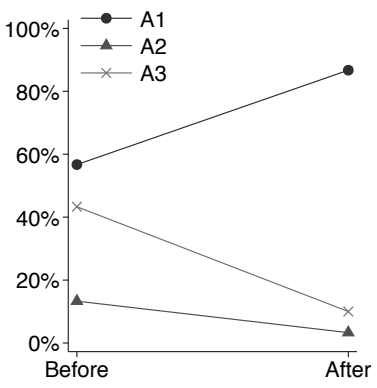

(a)

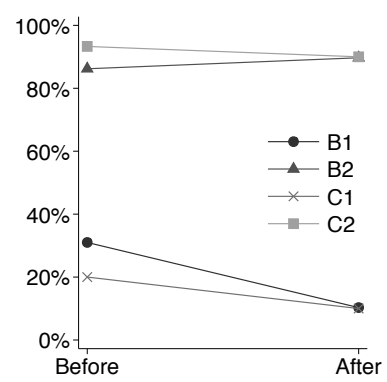

(b)
Figure 2: Percent of subjects favoring a design before and after performing (a) Task 1, and (b) Task 2 and Task 3.

ably within the task's context. Figure 2a illustrates how the favored design changed after performing Task 1 . While an orange-to-green gradient colors were generally considered to be a good idea, this particular color scheme was clearly harder to use in-context for finding lines with the most or the least knowledge, and this resulted in 9 of 11 supporters of design $A 3$ switching fully to $A 1$. Similarly, after performing Task 1, out of 4 subjects who initially favored the design with a different intensity of green, as in $(A 2), 2$ switched to design $A 1$ and 1 switched to design $A 3$.

The favored designs for the history-based annotation and recommendation of links also changed for some subjects after assessing the designs in the context of Tasks 2 and Task 3. Figure $2 \mathrm{~b}$ combines odd- and even-numbered subjects and shows the change in favored designs for annotating links with browsing history and recommendation. The number of subjects who favored design $B 2$ increased after performing Task 2 while the number of supporters for design $B 1$ decreased. Preference for recommendation designs changed as well. The number of subjects who favored design $C 1$ and $C 2$ decreased with the latter one experiencing less loss, only loosing one of its supporters.

Taken together, these results show that two or more alternatives for the selection of visual cues within the same conceptual ANS approach might differ significantly from the prospect of user perception and task performance. Moreover, user assessment of different ANS design options could considerably change when working with them in both a realistic context and in combinations with other visual cues. However, it was interesting that in all cases, the top designs $A 1-B 2-C 2$ identified in an out-of-context assessment increased their standing above other designs during in-context evaluation. Our findings stress the need to pay attention to designing visual cues, and not only to the approaches themselves. 


\section{REFERENCES}

[1] P. Brusilovsky. Adaptive navigation support. In The adaptive web, pages 263-290. Springer, 2007.

[2] P. Brusilovsky, J. Eklund, and E. Schwarz. Web-based education for all: A tool for developing adaptive courseware. In H. Ashman and P. Thistewaite, editors, Seventh International World Wide Web Conference, volume 30, pages 291-300. Elsevier Science B. V., 1998.

[3] P. Brusilovsky and M. V. Yudelson. From webex to navex: Interactive access to annotated program examples. Proceedings of the IEEE, 96(6):990-999, 2008.

[4] R. Hosseini, I.-H. Hsiao, J. Guerra, and P. Brusilovsky. What should i do next? adaptive sequencing in the context of open social student modeling. In 10th European Conference on Technology Enhanced Learning (EC-TEL 2015), pages 155-168, 2015.
[5] I. H. Hsiao, F. Bakalov, P. Brusilovsky, and B. Koenig-Ries. Progressor: social navigation support through open social student modeling. New Review of Hypermedia and Multimedia, 19(2):112-131, 2013.

[6] T. Loboda, J. Guerra, R. Hosseini, and P. Brusilovsky. Mastery grids: An open source social educational progress visualization. In 9th European Conference on Technology Enhanced Learning (EC-TEL 2014), volume 8719 of Lecture Notes in Computer Science, pages 235-248, 2014.

[7] K. A. Papanikolaou, M. Grigoriadou, H. Kornilakis, and G. D. Magoulas. Personalising the interaction in a web-based educational hypermedia system: the case of inspire. User Modeling and User Adapted Interaction, 13(3):213-267, 2003. 